\title{
A Patient with Giant Rippled-Pattern Sebaceoma in the Occipital Region
}

\author{
Masayuki Takahashi ${ }^{a} \quad$ Masaru Arima $^{a} \quad$ Yohei Iwata $^{a} \quad$ Kayoko Suzuki $^{b}$ \\ Yoshikazu Mizoguchic ${ }^{c}$ Makoto Kuroda ${ }^{d}$ Kayoko Matsunaga $^{a}$ \\ ${ }^{a}$ Department of Dermatology, Fujita Health University School of Medicine, \\ Toyoake, Japan; ${ }^{b}$ Department of Dermatology, Kariya Toyota General Hospital, \\ Kariya, Japan; ' Department of Diagnostic Pathology, Fujita Health University Banbuntane \\ Houtokukai Hospital, Nagoya, Japan; ${ }^{d}$ Department of Diagnostic Pathology, Fujita Health \\ University School of Medicine, Toyoake, Japan
}

\section{Keywords}

Rippled pattern · Giant sebaceoma - Occipital region

\section{Abstract}

A 72-year-old male visited a nearby hospital with a large tumor in his occipital region, which had existed since 20 years. Since malignant tumor was suspected, he was referred to our department. At the initial consultation, an elastic-hard, yellow-brown, sessile tumor, measuring $8 \times 7 \times 5 \mathrm{~cm}$ and with a flat surface, was observed in the occipital region. The tumor was resected and covered with artificial dermis. Histopathologically, the lesion was composed of basal-cell-like cells with nest formation in the dermis. A rippled pattern, or the single-line arrangement of tumor cells involving the stroma, was present. In addition, some tumor clusters revealed the differentiation to sebaceous glands, and these cells were positive for cytokeratin (AE1/AE3) and epithelial membrane antigen, which is consistent with the staining of sebaceous glands. On the contrary, tumor cells were negative for epithelial antigen (BerEP4), and Ki67 (MIB1) index was 5\% or lower. Therefore, we diagnosed the tumor as rippledpattern sebaceoma and not as basal cell carcinoma. Although this case was quite unique in its large size, immunostaining was useful for the definite diagnosis. 


\section{Introduction}

The rippled pattern is observed in about $25 \%$ of sebaceomas. The clinicopathological characteristics of rippled-pattern sebaceoma (RPS) are that it arises with a significant predominance in males and most frequently on the scalp, whereas sebaceoma without a rippled pattern arises on the face [1]. We report a case of giant RPS in the occipital region.

\section{Case Report}

The patient was a 72-year-old male with a history of hypertension. He consulted our hospital with a mass in the occipital region. His family history was non-contributory. He had hit his head on his garage roof 20 years prior to the initial consultation. Subsequently, a thumb-sized subcutaneous node developed on the occipital region and gradually expanded. He consulted the Department of Dermatology, Kariya Toyota General Hospital, with complaint of discomfort at bedtime and was referred to our department.

At the initial consultation, an elastic-hard, yellow-brown, sessile tumor, measuring $8 \times 7$ $\times 5 \mathrm{~cm}$ and with a flat surface, was detected in the occipital region (fig. 1). Palpation did not reveal any abnormalities in the bilateral cervical lymph nodes. A blood test did not show any abnormalities. On magnetic resonance imaging (MRI), the mass was visualized as a signal intensity similar to that of the gray-white matter. At the margin, a capsule-like structure was observed, showing low signal intensity. There was no cranial or intracranial infiltration. The clinical findings suggested an adnexal tumor involving the pilosebaceous or sweat-gland systems. The tumor was resected from an area $2 \mathrm{~cm}$ from the tumor base, and from the epicranial aponeurosis at the floor. The resected site was covered with artificial dermis. There has been no tumor relapse during the 39-month postoperative follow-up.

Histopathologically, proliferating alveolar cells were observed in the corium. In the tumor, a rippled pattern, or the single-line arrangement of basal-cell-like tumor cells involving the stroma, was present. Furthermore, differentiation to sebaceous glands was observed (fig. 2). There was no differentiation of hair bulbs/papilla to inferior hair follicles. Clefts between tumor lesion and stroma were only noted focally. On immunostaining, the resected specimen was positive for cytokeratin (AE1/AE3) and epithelial membrane antigen (EMA), consistent with the staining of sebaceous glands (fig. 3), and was negative for epithelial antigen (BerEP4). The Ki67 (MIB1) index was 5\% or lower.

\section{Discussion}

In the present patient, the tumor was large, and clefts were present between the alveolar tumor structure and stroma. It was necessary to differentiate the sebaceoma from appendage-derived malignant tumor and basal cell carcinoma (BCC). In sebaceoma, clefts between aggregations and surroundings stroma are rare and there is no palisaded arrangement of nuclei at the peripheries of the aggregations [2].

A rippled pattern was found within $25 \%$ of sebaceoma cases in one study. These tumors showed a male predominance and an affinity to arise on the scalp (as opposed to the face), when compared with sebaceomas, which lacked this growth pattern [3]. In contrast, the rippled pattern in BCC is relatively rare. In order to investigate the frequency of the rippled pattern in BCC, Misago et al. [4] reviewed 650 BCC specimens from the archives of their in- 
(C) 2016 The Author(s). Published by S. Karger AG, Basel www.karger.com/cde

Takahashi et al.: A Patient with Giant Rippled-Pattern Sebaceoma in the Occipital Region

stitution, and only one example with a rippled pattern was found among them. In addition, BCC rarely show follicular differentiation, and sebaceous differentiation is an exceedingly rare event in BCC [4].

Sebaceomas are rarely positive for Ber-EP4. Coupled with EMA to highlight the mature sebocytes, the use of Ber-EP4 and EMA is a reliable means of separating sebaceoma (BerEP4-/EMA+) from BCC (Ber-EP4+/EMA-) [5]. Based on the immunohistochemical analyses, we diagnosed the tumor as RPS not as BCC.

Sebaceous gland cancer is thought to arise de novo and not from benign sebaceous precursor lesions [6]. In theory, sebaceous carcinoma can occur anywhere where sebaceous glands are located. In practice, ocular sebaceous carcinoma is far more common than its extraocular counterpart, although both are aggressive and potentially lethal malignant neoplasms [7]. In the present case, the tumor was located in the occipital region and was slowly expanding. Based on an MIB1 index of $<5 \%$, the tumor was evaluated as benign. We believe that the possibility of future malignancy was low.

The tumor may have enlarged in the absence of treatment and in the presence of chronic stimuli, such as clothing-related compression and friction. A search of RPS sizes on PubMed suggested that the tumor of the present patient was the largest. Histopathologically, it was necessary to make a differential diagnosis from BCC due to the differentiation of sebaceous glands, and immunostaining allowed for a differential diagnosis.

\section{Statement of Ethics}

The patient gave written informed consent.

\section{Disclosure Statement}

The authors have no conflicts of interest.

\section{References}

1 Ansai S, Kimura T: Rippled-pattern sebaceoma: a clinicopathological study. Am J Dermatopathol 2009;31:364-366.

2 Troy JL, Ackerman AB: Sebaceoma. A distinctive benign neoplasm of adnexal epithelium differentiating toward sebaceous cells. Am J Dermatopathol 1984;6:7-13.

3 Biswas A, Setia N, Bhawan J: Cutaneous neoplasms with prominent Verocay body-like structures: the so-called 'rippled pattern'. Am J Dermatopathol 2011;33:539-548.

4 Misago N, Tsuruta N, Narisawa Y: Rippled-pattern basal cell carcinoma. J Dermatol 2012;39:632-635. Fan YS, Carr RA, Sanders DS, Smith AP, Lazar AJ, Calonje E: Characteristic Ber-EP4 and EMA expression in sebaceoma is immunohistochemically distinct from basal cell carcinoma. Histopathology 2007;51:80-86.

6 Rizvi M, De Jesus R, Girotto J, Nonaka D, Goldberg N: Sebaceous gland carcinoma of the scalp: case report and review of the literature. J Otolaryngol 2003;32:64-68.

7 Crowson AN, Magro CM, Mihm MC: Malignant adnexal neoplasms. Mod Pathol 2006;19(suppl 2):S93S126. 


\section{Case Reports in
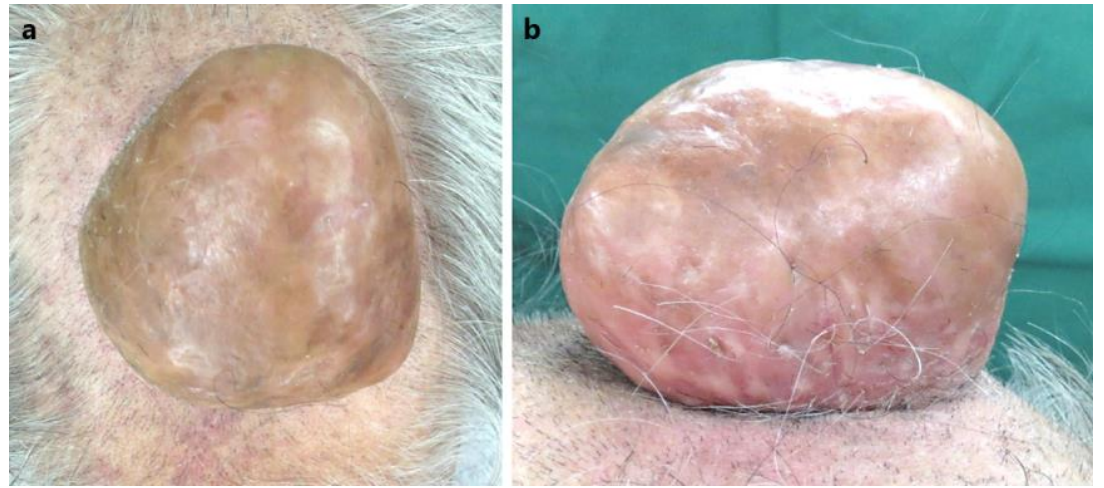

Fig. 1. The elastic-hard, yellow-brown (b), sessile tumor, measuring $8 \times 7 \times 5 \mathrm{~cm}$, with a flat surface in the occipital region (a).
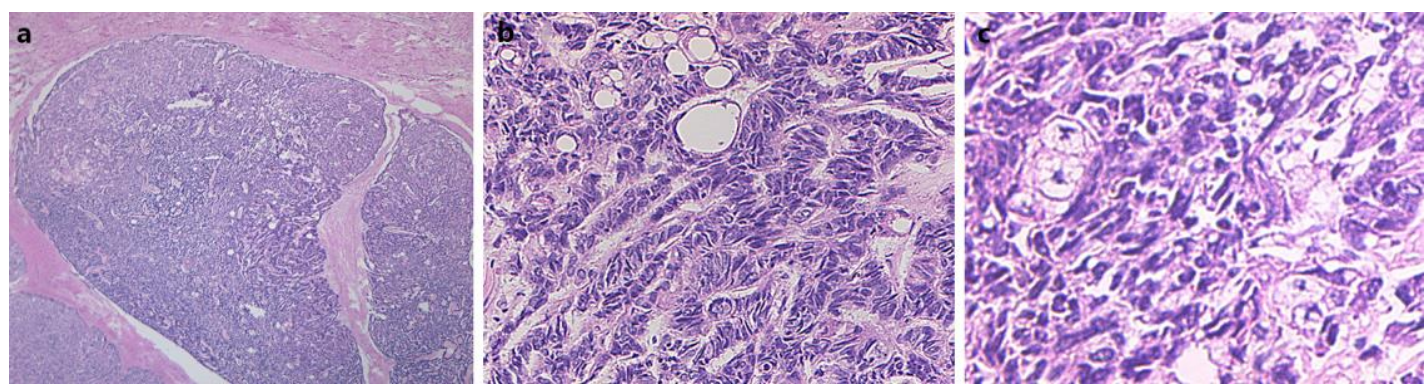

Fig. 2. Proliferating alveolar cells observed in the corium. a Partial clefts were noted. A rippled-pattern (b) and differentiation to sebaceous glands were observed in the tumor (c). 


\section{Case Reports in
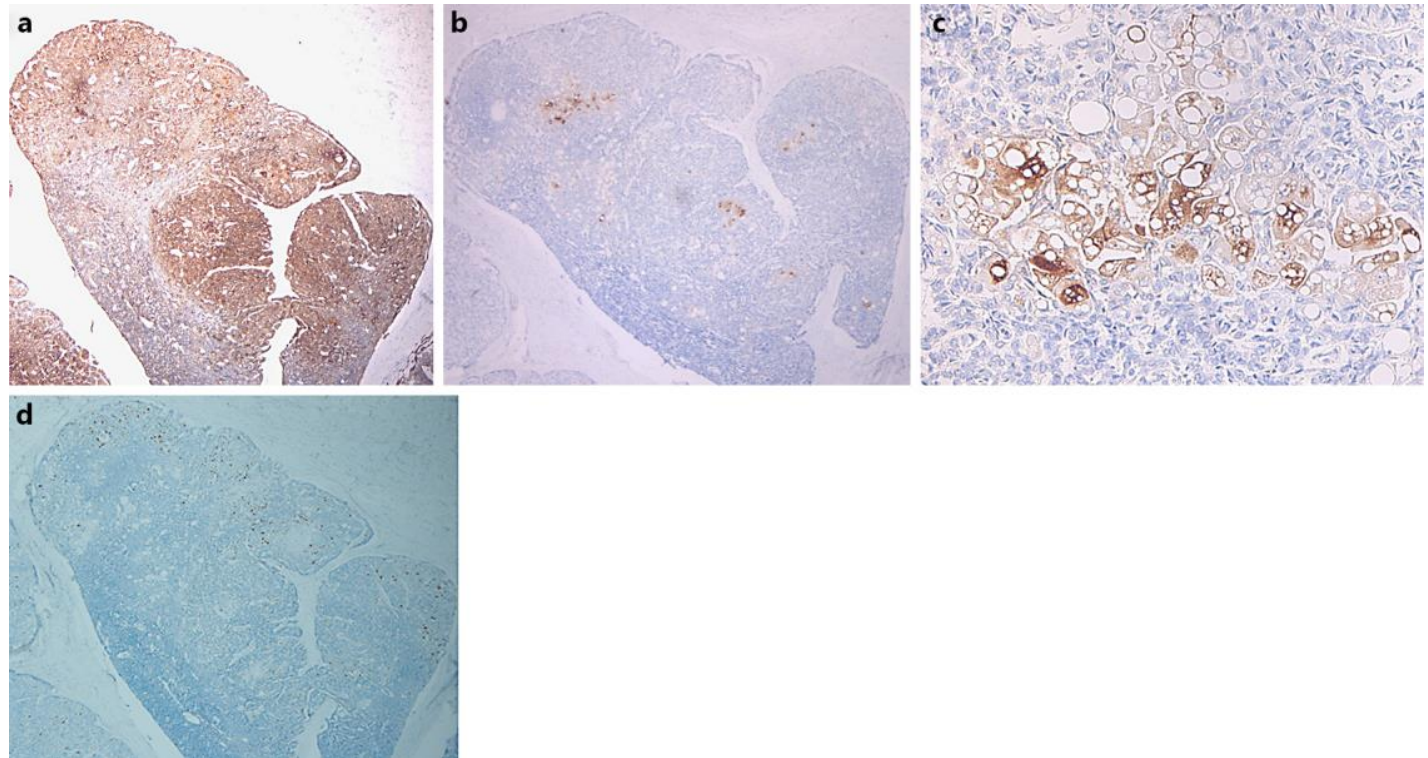

Fig. 3. a The resected specimen was positive for AE1/AE3. b The tumor was also positive for EMA, consistent with sebaceous glands. $\times 40$. c EMA staining: magnified view. $\times 100$. $d$ The MIB1 index was $5 \%$ or lower. $\times 40$. 